\title{
Journal-club pour le praticien Journal-Klub für den Praktiker
}

\author{
Une sélection du journal-club pour le praticien \\ de la Clinique de Genève/J.-H. Saurat \\ Eine Auswahl des Journal-Klubs für den Praktiker \\ der Genfer Klinik/J.-H. Saurat
}

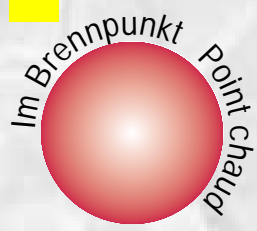

\section{Code postal: peau - \\ Ou comment certains \\ lymphocytes migrent dans la peau et pas ailleurs}

[Nat Med 2002;7:157]

Dermatite atopique, psoriasis et dermite allergique de contact, ces «trois top models» de la dermatologie inflammatoire, sont induits par des lymphocytes $\mathrm{T}$ activés ou de mémoire qui infiltrent spécifiquement la peau. On pense que ces lymphocytes reviennent dans la peau parce qu'après y avoir été éduqués, ils y sont spécifiquement attirés et retenus. Pour cela il faut deux choses: une «adresse» sur le lymphocyte, et une «boîte postale» dans la peau correspondant à cette adresse. On en sait maintenant beaucoup plus sur ces deux éléments. Tout d'abord l'adresse sur le lymphocyte: le code général «peau» (comme 3000 pour Berne) est CLA («cutaneous lymphocyte-associated antigen», une molécule d'adhésion) que portent $15 \%$ des lymphocytes qui circulent dans le sang; les codes secondaires sont CCR4 et CCR10 (comme 3006 pour la Beatusstrasse à Berne et 3800 pour Interlaken), des récepteurs de chémokines que portent seulement une partie des lymphocytes CLA+. Les lymphocytes acquièrent ces «codes postaux» lors de leur activation dans les ganglions lymphatiques qui drainent la peau (les codes de l'intestin, différents de ceux de la peau, sont acquis dans les ganglions qui drainent l'intestin). Ainsi, activés par un pathogène à la porte d'entrée cutanée, ils vont naturellement avoir l'adresse pour revenir dans la peau, où ce pathogène risquerait de se représenter, et pas ailleurs. Voilà pour le code postal sur les lymphocytes, voyons maintenant la boîte postale dans la peau. Elle se compose d'abord d'une molécule appelée Esélectine qui est exprimée sur l'endothélium des vaisseaux et qui se lie à CLA, retenant ainsi tous les lymphocytes CLA+ qui circulent. Elle se compose ensuite de chémokines, CCL17 et CCL27, sécrétées par les kératinocytes, qui vont se fixer sur leurs récepteurs, CCR4 et CCR10 respectivement, et ainsi attirer une partie des lymphocytes CLA+. Ainsi un lymphocyte dont le code est CLA+/CCR4+/CCR10+ sera délivré dans la boîte postale E-sélectine+/ CCL17+/CCL27+. Il s'en suivra une réaction inflammatoire spécifique et compartimentalisée, par exemple un eczéma de contact, une poussée de dermatite atopique ou un psoriasis en gouttes... L'article de Nature Medicine porte sur la paire CCR10/CCL27; il montre que la plupart des lymphocytes présents dans la peau des dermatites atopiques, dermites allergiques de contact et psoriasis sont CCR10+, et que CCL27 est très exclusivement exprimée dans la couche basale de l'épiderme, diffuse et colle à la matrice extracellulaire et à l'endothélium, ce qui fait de cette chémokine une «boîte postale» très spécifique de la peau. Ainsi en inhibant spécifiquement CCL27, on devrait pouvoir obtenir un effet immunosuppresseur «compartimentalisé» à la peau. C'est ce que montrent les auteurs de l'article: ils inhibent chez la souris des réactions allergiques avec un anticorps anti-CCL27 d'une façon plus efficace qu'avec le FK506. On entrevoit ainsi une nouvelle ère dans le traitement de ces maladies cutanées: l'inhibition spécifique de la migration dans la peau des lymphocytes porteurs du code postal «peau», en enlevant simplement l'adresse de la boîte aux lettres! Reste à savoir où ira le courrier non délivré!

\section{Destination Haut - Oder weshalb gewisse Lymphozyten in die Haut und nicht anderswohin wandern}

[Nat Med 2002; 7:157]

Neurodermitis, Psoriasis, allergisches Kontaktekzem - diese drei «Leitbilder» unter den entzündlichen Hautkrankheiten werden durch aktivierte (Memory-)T-Lymphozyten ausgelöst, die spezifisch in die Haut eindringen. Man nimmt an, dass diese Lymphozyten zur Haut zurückkehren, weil sie dort herangebildet wurden und deshalb von der Haut spezifisch angezogen und zurückgehalten werden. Bildlich gesprochen sind hierzu folgende zwei Voraussetzungen erforderlich: eine "Adressangabe» auf dem Lymphozyten und ein «Briefkasten» in der Haut, der dieser Adresse entspricht. Heute weiss man über diese Bedingungen sehr viel besser Bescheid. Erstens über die Adresse auf den Lymphozyten: Die allgemeine «Postleitzahl» (wie 3000 für Bern) lautet CLA («cutaneous lymphocyte-associated antigen»); hierbei handelt es sich um ein Adhäsionsmolekül, das $15 \%$ der zirkulierenden Lymphozyten tragen. Die ortsspezifischen Postleitzahlen (wie 3006 für Bern Beatusstrasse und 3800 für Interlaken) lauten CCR4 und CCR 10; dies sind Chemokinrezeptoren, die nur auf gewissen CLA-positiven Lymphozyten vorhanden sind. Die Lymphozyten erhalten diese «Postleitzahlen» bei ihrer Aktivierung in den Lymphknoten, welche die Lymphe aus der Haut abführen. Analog dazu werden darmspezifische «Leitzahlen» von Lymphknoten erteilt, die den Darm drainieren. Werden Lymphozyten also durch einen Erreger aktiviert, der durch die Haut eindringt, wandern sie aufgrund ihrer «Adressierung» gleichsam automatisch dorthin, wo dieser Krankheitskeim am wahrscheinlichsten in Erscheinung tritt. Soviel zur Postleitzahl auf den Lymphozyten; werfen wir nun einen Blick auf den Briefkasten in der Haut. Dazu gehört als erstes ein Molekül mit der Bezeichnung E-Selektin, das auf dem Gefässendothel exprimiert wird und an CLA bindet. Dadurch werden alle CLA-positiven Lymphozyten aus dem Kreislauf herausgegriffen. Weitere Bestandteile des Briefkastens sind die Chemokine CCL17 und CCL27, die von den Keratinozyten sezerniert werden und an die entsprechenden Rezeptoren, CCR4 
bzw. CCR10, binden, um anschliessend einen Teil der CLA-positiven Lymphozyten anzuziehen. Demzufolge wird ein Lymphozyt, dessen Postleitzahlen CLA+/ CCR4+/CCR10+ lauten, in den Briefkasten E-Selektin+/CCL17+/CCL27+ «eingeworfen» werden. Anschliessend kommt es zu einer spezifischen und abgegrenzten entzündlichen Reaktion beispielsweise einem Kontaktekzem, einem Neurodermitisschub oder einer Psoriasis guttata. Im vorliegenden Artikel aus «Nature Medicine» wird das Paar CCR10/CCL27 besprochen. Es wird aufgezeigt, dass die Mehrzahl der bei Neurodermitis, Kontaktekzem und Psoriasis in der Haut vorhandenen Lymphozyten CCR10-positiv sind und dass CCL27 ausschliesslich in der Basalmembran der Epidermis exprimiert wird, in die extrazelluläre Matrix und das Endothel diffundiert und dort haften bleibt. Dadurch wird dieses Chemokin zu einem sehr spezifischen «Briefkasten» der Haut. Durch spezifische Hemmung von CCL27 sollte es daher möglich sein, einen auf die Haut begrenzten immunsuppressiven Effekt zu erzielen. Genau dies zeigen die Verfasser des Artikels auf: Sie hemmen bei der Maus allergische Reaktionen mit Hilfe eines Anti-CCL27-Antikörpers auf wirksamere Weise, als dies mit FK506 möglich ist. Dies lässt neue Behandlungsmöglichkeiten für diese Hauterkrankungen erahnen: die gezielte Hemmung der Migration von Lymphozyten, die an die Haut «adressiert» sind, indem ganz einfach der Name des Empfängers vom Briefkasten entfernt wird! Bleibt abzuwarten, wo die unzustellbare Postsendung schliesslich landet...

\section{La lipodystrophie des traitements anti-VIH: une histoire de transcription}

[Lancet 2002;359:1026]

Les traitements associant les inhibiteurs de protéases et de transcriptases reverses ont amélioré le pronostic de l'infection par le VIH, mais de nombreux patients développent des troubles métaboliques (dyslipidémie et résistance à l'insuline) et une lipodystrophie avec lipoatrophie périphérique. Cette anomalie de distribution des graisses est en rapport avec un trouble de la différenciation des adipocytes. De nombreuses théories, plus ou moins fondées, ont été proposées ces dernières années pour expliquer comment les anti-VIH (surtout les antiprotéases) pouvaient induire un trouble de la différenciation des adipocytes et ainsi une lipodystrophie. Certains avaient soupçonné le métabolisme des rétinoïdes. Plus récemment on s'est orienté vers un système voisin, celui des PPAR- $\gamma$ (voir Dermatologica Helvetica 2/2002) qui jouent un rôle important dans le métabolisme des graisses. Des nutritionistes parisiens fournissent maintenant des données d'importance pratique. Ils ont prélevé du tissu adipeux par liposuccion chez les patients infectés par le VIH et traités par les inhibiteurs de protéases et de transcriptases reverses; la liposuccion était destinée à combler la lipoatrophie du visage, et une partie du prélèvement a servi à des études morphologiques et moléculaires. Au plan morphologique, les adipocytes étaient de petite taille et pouvaient correspondre à des cellules jeunes non différenciées. Au plan moléculaire, les facteurs de transcription qui commandent la sensibilité à l'insuline et la différenciation des adipocytes (PPAR- $\gamma, \mathrm{C} / \mathrm{EBP}, \mathrm{SREBP} 1$ ) étaient très diminués dans la graisse de ces patients; les auteurs confirment ainsi in vivo ce qu'ils avaient observé in vitro, à savoir que les antiprotéases inhibent SREBP1, un facteur de transcription déterminant dans ce système. Ces observations ont un intérêt pratique; en effet, les glitazones, une nouvelle classe d'hypoglycémiants, qui se lient aux PPAR- $\gamma$ pourraient constituer un traitement médical, peut-être préventif, de ces lipodystrophies.

\section{Lipodystrophie bei der Behandlung mit Anti-HIV- Medikamenten: eine Frage der Transkription}

[Lancet 2002;359:1026]

Die Kombinationstherapie mit Protease- und Reverse-Transkriptase-Hemmern hat die Prognose von Patientinnen und Patienten mit einer HIV-Infektion verbessert. Bei vielen von ihnen treten jedoch Stoffwechselstörungen (Dyslipidämie und Insulinresistenz) sowie eine Lipodystrophie mit peripherer Lipatrophie auf. Diese Anomalie bei der Fettverteilung steht in Zusammenhang mit einer gestörten Differenzierung der Adi- pozyten. Zahlreiche - mehr oder weniger fundierte - Theorien sind in den letzten Jahren aufgestellt worden, um zu erklären, wie Anti-HIV-Medikamente (insbesondere Proteasehemmer) Störungen der Fettzelldifferenzierung und damit eine Lipodystrophie bewirken können. Einzelne Autoren verdächtigten in diesem Zusammenhang den Retinoidstoffwechsel. In letzter Zeit hat man sich vermehrt mit dem damit verwandten System der PPAR- $\gamma$ beschäftigt (siehe «Dermatologica Helvetica» 2/2002), die im Fettstoffwechsel eine wichtige Rolle spielen. Ernährungsforscher aus Paris warten jetzt mit praxisrelevanten Daten auf. Sie haben bei HIV-infizierten Patienten mittels Liposuktion Fettgewebsproben entnommen und diese mit Protease- und Reverse-TranskriptaseHemmern behandelt. Die Liposuktion war zur Behebung der Lipatrophie im Gesicht durchgeführt worden, und ein Teil des entnommenen Gewebes wurde anschliessend für morphologische und molekulare Untersuchungen verwendet. Aus morphologischer Sicht wiesen die Adipozyten eine geringe Grösse auf und entsprachen jungen, undifferenzierten Zellen. Auf molekularer Ebene waren die Transkriptionsfaktoren, welche die Insulinempfindlichkeit und Differenzierung der Adipozyten steuern (PPAR- $\gamma$, C/EBP, SREBP1), im Fettgewebe dieser Patienten stark vermindert. Die Autoren bestätigen damit in vivo ihre vorangehenden Beobachtungen in vitro, nämlich dass Proteasehemmer SREBP1, einen entscheidenden Transkriptionsfaktor in diesem System, hemmen. Diese Resultate sind von praktischer Bedeutung: Die Glitazone - eine neue Klasse blutzuckersenkender Medikamente -, die an PPAR- $\gamma$ binden, könnten sich nämlich zur medikamentösen Therapie und eventuell Prophylaxe dieser Lipodystrophien eignen.

\section{Ce nickel que I'on respire en Russie}

[J Am Acad Dermatol 2002;46:460]

La prévalence de l'allergie cutanée au nickel avoisine les 20\% dans les pays de l'Europe occidentale, surtout nordique. On pense généralement que cette forte prévalence est due à une exposition importante, que les pièces d'Euro vont en- 
core augmenter (elles contiendraient plus de nickel que l'autorise la commission ad hoc de Bruxelles...). En Russie, la pollution atmosphérique par le nickel est très élevée dans des villes bien nommées de Nikel et Zapolyarny (près de Mourmansk), sièges d'usines de raffinage. On pourrait donc penser que la prévalence de l'allergie au nickel dans ces deux villes est très élevée; une étude conduite par une équipe d'épidémiologistes norvégiens et russes démontre le contraire; les tests épicutanés au nickel ne sont positifs que chez 7\% des 657 habitantes de Nikel et Zapolyarny testés, alors qu'ils le sont chez $27 \%$ des 690 voisines norvégiennes. Les percements d'oreilles étaient observés chez le même pourcentage dans les deux groupes, soit $85 \%$. Les auteurs suggèrent que l'exposition à la pollution au nickel pourrait induire une tolérance immunitaire pour cet haptène. De là à conseiller le doux climat de Nikel et Zapolyarny...

\section{Nickelbelastung der Luft in Russland}

[J Am Acad Dermatol 2002;46:460]

Die Häufigkeit von Hautallergien gegen Nickel beläuft sich in Westeuropa insbesondere im Norden - auf rund 20\%. Man geht im Allgemeinen davon aus, dass dieser beträchtliche Prozentsatz von einer hohen Exposition herrührt, die übrigens durch die Euro-Münzen noch verstärkt werden dürfte (sie sollen mehr Nickel enthalten, als die Ad-hoc-Kommission in Brüssel für zulässig erachtet). In Russland ist die atmosphärische Belastung durch Nickel in den Städten Nikel und Zapolyarny in der Nähe von Murmansk, in denen Aufbereitungsanlagen betrieben werden, besonders hoch. Man würde daher annehmen, dass Nickelallergien in diesen Städten besonders häufig vorkommen. Eine durch norwegische und russische Epidemiologen durchgeführte Studie hat aber das Gegenteil ergeben: Epikutanproben auf Nickel fielen nur gerade bei 7\% der 657 untersuchten Einwohnerinnen von Nikel und Zapolyarny positiv aus, während dies bei $27 \%$ von 690 getesteten Einwohnerinnen aus dem benachbarten Norwegen der Fall war. Beide Gruppen von Frauen wiesen mit 85\% die gleiche Quote durchstochener Ohrläppchen auf. Die Autoren vermuten, dass die Exposition gegenüber der nickelbelasteten Luft eine Immuntoleranz für dieses Hapten bewirken könnte. Diesbezüglich wäre das «milde Klima» von Nikel und Zapolyarny zu empfehlen...

\section{Nouvelles de la SSDV Neues aus der SGDV}

\section{Distinctions \\ Auszeichnungen - Ehrungen Onoranze}

Anlässlich der Mitgliederversammlung der Deutschen Gesellschaft für Allergologie und klinische Immunologie am 14. März 2002 in Bochum wurde Prof. B. Wüthrich wegen «seiner grossen Verdienste um die Allergologie und klinische Immunologie» mit der Ehrenmitgliedschaft der DGAI ausgezeichnet.

Anlässlich des Kongresses im Mai 2001 wurde Prof. B. Wüthrich die Ehrenmitgliedschaft der Foundation for Allergy Research in Europe (FARE) verliehen.

Anlässlich der Jahresversammlung der Schweizer Gesellschaft für Allergologie und Immunologie vom 14. und 15. März 2002 in Lugano wurde das Poster «Component-resolved diagnosis using recombinant allergens in cherry-allergic patients» von B.K. Ballmer-Weber, S. Scheurer, P. Fritsche, B. Wüthrich, E. Enrique, A. Cistero-Balhima, T. Haase und S. Vieths mit dem FerdinandWortmann-Poster-Preis für Allergologie ausgezeichnet. Frau Dr. Ballmer-Weber ist Oberärztin auf der Allergiestation und hat ein Stipendium der Universität Zürich zur Förderung des akademischen Nachwuchses (Stiefel-Zangger-Stiftung).
Nouveau cabinet médical Praxiseröffnung Nuovo studio medico

\section{Urs Bader}

Facharzt FMH für Dermatologie und Venerologie

Geissacher 6

8126 Zumikon

\section{Attribution du titre de médecin spécialiste \\ Verleihung des Spezialarzttitels \\ Conferimento del titolo di dottore specialista}

\section{Suzanne Freund-Kissling}

Fachärztin FMH für Dermatologie und Venerologie

Susenbergstrasse 104

8044 Zürich

\section{Daniel A. Fuchs}

Facharzt FMH für Dermatologie und Venerologie

Mittelfeldstrasse 7

8700 Küsnacht 